\title{
On the structure of the family of Cherry fields on the torus
}

\author{
COLIN BOYD \\ SES 5.2.2, MLB 5/56, B.T.R.L., Martlesham Heath, Ipswich 1P5 7RE, England
}

(Received 17 January 1984)

Abstract. A class of vector fields on the 2-torus, which includes Cherry fields, is studied. Natural paths through this class are defined and it is shown that the parameters for which the vector field is unstable is the closure of $\left\{t \mid R_{t} \circ f\right.$ has irrational rotation number $\}$, where $f$ is a certain map of the circle and $R_{t}$ is rotation through $t$. This is shown to be a Cantor set of zero Hausdorff dimension. The Cherry fields are shown to form a family of codimension one submanifolds of the set of vector fields. The natural paths are shown to be stable paths.

\section{Introduction and statement of results}

We are interested in certain flows of class $C^{\infty}$ on the 2-torus. We will work on its universal cover $\mathbb{R}^{2}$, so all vector fields $X$ will satisfy $X(x+n, y+m)=X(x, y)$, for all $n, m \in \mathbb{Z}$. All the vector fields considered will satisfy the following:

(A) $X$ has two singularities, a hyperbolic saddle $S$ and a hyperbolic sink $P$.

(B) $X$ is transverse to the circle $\Sigma=\{(x, y) \mid x=0\}$.

(C) There exist $a, b \in \Sigma$ such that if $y \in(a, b)$ the positive orbit of $X$ through $y$ goes directly to the sink without re-intersecting $\Sigma$, but for $y \notin[a, b]$ the Poincaré map $f: \Sigma \rightarrow \Sigma$ is defined and expanding. Furthermore, $f^{\prime}(y) \rightarrow \infty$ as $y \rightarrow a^{-}$or $y \rightarrow b^{+}$. (See figure 1a.)
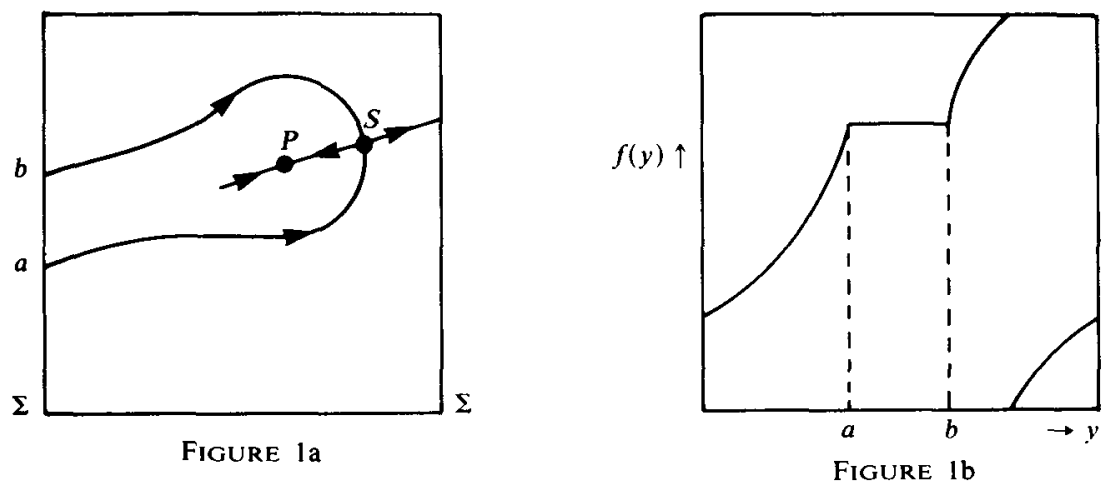

The Poincaré map may be extended to the whole of $\Sigma$ by making it constant on $[a, b]$ so we have a continuous circle endomorphism $f: \Sigma \rightarrow \Sigma$. By condition (C), $f^{\prime}(y) \geq \lambda>1$ for all $x \notin[a, b]$. (See figure $1 \mathrm{~b}$.)

Since $f$ is monotonic and of degree one it has a rotation number (see e.g. [8]). We denote the set of $C^{\infty}$ vector fields on the 2-torus with the $C^{\infty}$ topology, by 
$\mathfrak{X}^{\infty}\left(T^{2}\right)$, and the neighbourhood in $\mathfrak{X}^{\infty}\left(T^{2}\right)$ of all vector fields satisfying (A), (B) and $(\mathrm{C})$, by $\mathcal{N}$.

(1.1) Definition. A Cherry field is a vector field in $\mathcal{N}$ whose Poincaré map has irrational rotation number.

Cherry fields were first constructed in [3]; see [8, pp. $181 \mathrm{ff}]$ for a modern construction. The orbit structure of a Cherry field is described by the following:

(1.2) TheOREM ([8], p. 186). Let $X$ be a Cherry field with sink $P$ and saddle $S$. Then

(1) $W^{s}(P)$ is dense in $T^{2}$.

(2) $P$ and $S$ are the only minimal sets for $X$.

(3) $\Sigma-W^{s}(P)$ is a Cantor set.

(4) $T^{2}-W^{s}(P)$ is transitive for the flow.

Vector fields $X \in \mathcal{N}$ whose Poincaré maps have rational rotation number are either Morse-Smale or have a saddle connection, the fields with a saddle connection forming the boundary of the Morse-Smale classes with the same rotation number. The three types of field in $\mathcal{N}$ are determined by what happens to the orbit of the 'free' unstable separatrix of $S$ - that not joined directly to the sink. One of the following must happen:

(i) after intersecting $\Sigma$ a number of times it intersects $(a, b)$ and goes to the sink. In this case $X$ is a Morse-Smale field;

(ii) after intersecting $\Sigma$ a number of times it intersects $\Sigma$ at $a$ or $b$, so $X$ has a saddle connection;

(iii) it intersects $\Sigma$ infinitely often without intersecting $[a, b]$. In this case $X$ is a Cherry field.

We investigate paths in $\mathcal{N}$ which change the relative positions of the free separatrix of $W^{u}(s)$ and $[a, b]$. By measuring how many parameter values correspond to Cherry fields we get an idea of how common they are in $\mathcal{N}$. Let $\phi:[0,1] \rightarrow \mathcal{N}$ be a $C^{1}$ path chosen so that

$$
f_{\phi(t)}(y)=f_{\phi(0)}(y)+t
$$

where $f_{\phi(t)}$ is the Poincaré map of $\phi(t)$. Such a path may be constructed by making a suitable perturbation to the vector field in a small strip near $\Sigma$.

Every number in $[0,1)$ is represented as the rotation number of $f_{\phi(t)}$ for some $t$, and it is not difficult to see that the bifurcation set of $\phi$ is a Cantor set $E$. The open intervals in the complement of $E$ consist of parameters corresponding to MorseSmale fields, the boundary points of these intervals correspond to fields with a saddle connection and the remaining points of $E$ correspond to Cherry fields. Our first result reveals that this path contains very few Cherry fields. Let $m$ denote Lebesgue measure.

(1.3) Theorem 1. Let $E=\{t \mid \phi$, is unstable $\}$. Then $m(E)=0$ and furthermore $E$ has zero Hausdorff dimension.

From well-known work of Sotomayor [10] it is known that the set of fields in $\mathcal{N}$ with a saddle connection forms an immersed submanifold of $\mathcal{N}$ of class $C^{\infty}$ and 
codimension one, or those with a particular rotation number form an embedded submanifold. We are able to show the following for Cherry fields:

(1.4) THEOREM 2. The set of Cherry fields in $\mathcal{N}$ with a given rotation number forms a codimension one embedded Banach submanifold of $\mathcal{N}$ of class $C^{\prime}$.

Note that the set of all Cherry fields is not an embedded submanifold, since there would be uncountably many components in any neighbourhood. We have no reason to believe that the submanifold is not, in fact, of class $C^{\infty}$. Using theorem 2 we prove the following about the path $\phi$ described above, which shows it is not a particularly special path.

(1.5) THEOREM 3. The path $\phi$ is stable in the space of $C^{1}$ paths in $\mathcal{N}$, as long as $\phi(0)$ is Morse-Smale.

$\phi$ is unusual as a stable path because the Cherry fields are Kupka-Smale but not Morse-Smale (see [10, p. 45]).

\section{Proof of theorem 1}

Theorem 1 will be implied by the slightly stronger:

(2.1) TheOREM 1'. Let $f$ be a continuous monotonic non-decreasing map of the circle of degree one satisfying

(1) $f$ is constant on an interval $[a, b]$ and of class $C^{1}$ outside $[a, b]$.

(2) $\inf \left\{f^{\prime}(y) \mid y \notin[a, b]\right\}=\lambda>1$.

Let $f_{t}$ be the map defined by $f_{t}(y)=f(y)+t, t \in[0,1]$. Let $E=\left\{t \mid f_{t}\right.$ has irrational rotation number $\}$. Then $m(E)=0$, where $m$ denotes Lebesgue measure, and furthermore $E$ has zero Hausdorff dimension.

Since the Poincare map of any field in $\mathcal{N}$ satisfies the hypotheses of theorem 1 ', it is clear that theorem $\mathbf{1}^{\prime}$ implies theorem 1 . Note that it does not matter in theorem 1 whether or not we include in $E$ the parameter values corresponding to saddle connections, since there are only countably many of them. Theorem 1' shows a contrast with the situation for diffeomorphisms of the circle, by comparison with the following:

(2.2) Theorem (Herman [5]). Let $f_{t}, t \in[0,1]$, be a $C^{1}$ path in the space of $C^{r}$ diffeomorphisms of $S^{1}$ with the $C^{r}$ topology, $r \geq 3$. Let $E=\left\{t \mid f_{t}\right.$ is $C^{r-2}$ conjugate to an irrational rotation\}. As long as the rotation number changes at all along the path, then $m(E)>0$.

(2.3) Proof of theorem 1'. We will assume, without loss of generality, that $a=0$, i.e. $f$ is constant on $[0, b], 0<b<1$, and that $f(0)=0$ (see figure $2 \mathrm{a}$ ). Consider the set $A \subset S^{1} \times[0,1]$ defined by

$$
A=\left\{(y, t) \mid y \in f_{i}^{-n}[0, b] \text { for some } n \geq 0\right\} .
$$

(See figure 2b.) We write

$$
A_{y}=\{t \mid(y, t) \in A\}, \quad A^{t}=\{y \mid(y, t) \in A\} .
$$




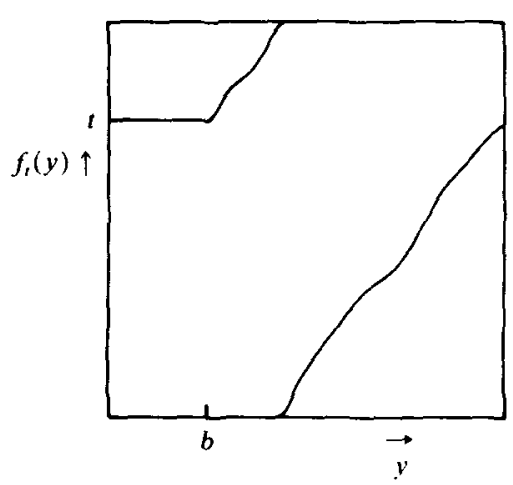

FIGURE 2a

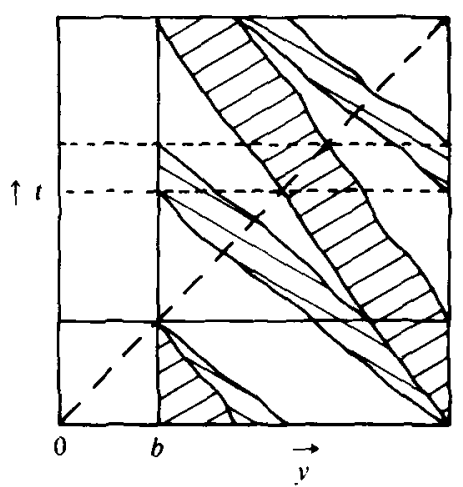

Figure 2b

Now

$$
\begin{aligned}
{[0,1]-E } & =\left\{t \mid f_{t} \text { has a periodic point }\right\} \\
& =\{t \mid t \text { is periodic }\} \quad \text { since } t \text { is always in the periodic orbit } \\
& =\left\{t \mid f_{t}^{n}(b) \in[0, b] \quad \text { for some } n \geq 1\right\} \quad \text { since } f_{t}(b)=t \\
& =A_{b}^{\prime}
\end{aligned}
$$

where for $y \in S^{1}$ we write

$$
A_{y}^{\prime}=\left\{t \mid y \in f_{t}^{-n}[0, b] \text { for some } n \geq 1\right\}
$$

Note that $A_{y}^{\prime}=A_{y}$ when $y \notin[0, b]$. We will show that in fact $m\left(A_{y}\right)=1$ for all $y \in S^{\prime}$. The first step is

(2.4) LEMMA. $m\left(A^{t}\right)=1$ for all $t \in[0,1]$.

Proof. Fix $t \in[0,1]$. We consider two distinct cases.

Case 1. $f_{t}^{-i}([0, b])$ intersects $f_{t}^{-j}[0, b]$ for some $i \neq j$. In this case $f_{t}$ has a periodic point of period $n=|i-j|$. Consider the graph of $f_{t}^{n}$. This has $n$ constant intervals separated by $n$ intervals where $\left(f_{t}^{n}\right)^{\prime}(y)>1$. Hence $f_{t}$ has exactly one attracting periodic orbit and one repelling periodic orbit. The attracting periodic orbit includes a point in $[0, b]$. Hence all but finitely many points end up in $[0, b]$ and so $\bigcup_{n=0}^{\infty} f_{t}^{-n}([0, b])$ has measure one; that is $m\left(A^{t}\right)=1$.

Case 2. $f_{t}^{-i}[0, b] \cap f_{t}^{-j}[0, b]$ is empty when $i \neq j$. It follows that

$$
\left|f_{t}^{-n}([0, b])\right| \leq b / \lambda^{n} \rightarrow 0 \quad \text { as } n \rightarrow \infty
$$

since $\lambda>1$. Now suppose for a contradiction that $m\left(\bigcup_{n=0}^{\infty} f_{t}^{-n}([0, b])\right)<1$. Let us write $a_{n}=\sum_{i=0}^{n}\left|f_{t}^{-i}[0, b]\right|$. Then the monotonic sequence $\left\{a_{n}\right\}_{n=1}^{\infty} \rightarrow l$ for some $l<1$. To each $f_{t}^{-i}[0, b], i \geq 1$, there corresponds a section of the graph of $f$ of height $\left|f_{t}^{-i-1}([0, b])\right|$ and length $\left|f_{i}^{-i}([0, b])\right|$ (see figure 3 ). Choose $N$ so large that $\left(1-a_{N}\right) /\left(1-a_{N+1}\right)<\lambda$, which is possible since $\left(1-a_{N}\right) /\left(1-a_{N+1}\right) \rightarrow 1$ as $N \rightarrow \infty$. After removing the sections of the graph of $f$ corresponding to $f_{t}^{-i}([0, b]), 0 \leq i \leq N$, there remain at most $N+2$ sections of total length $1-a_{N+1}$ and total height $1-a_{N}$. 


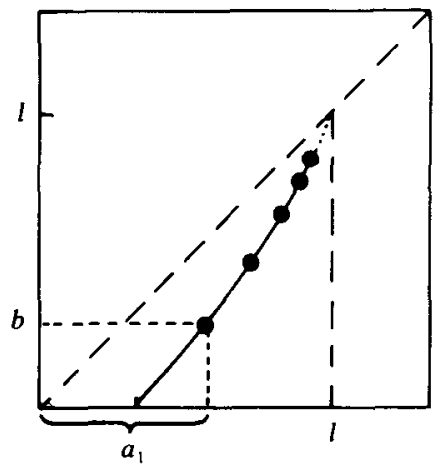

FIGURE 3

Since on each of these sections $f^{\prime}(y) \geq \lambda$, by the Mean Value Theorem $\left(1-a_{N}\right) /\left(1-a_{N+1}\right) \geq \lambda$. This contradiction completes the proof of (2.4).

(2.5) Remark. In particular, the Cantor set $\Sigma-W^{s}(P)$ mentioned in (1.2) has zero Lebesgue measure. This is because it is a set $A^{t}$ for some $t$ in case 2. This Cantor set also has zero Hausdorff dimension by arguments similar to those below using (2.7).

(2.6) LeMMA. $m\left(A_{y}^{\prime}\right)$ is a continuous function of $y$.

Proof. Consider the functions $f_{y, n}(t):[0,1] \rightarrow S^{1}$ defined by $f_{y, n}(t)=f_{t}^{n}(y), n \geq 1$. Thus

$$
\begin{gathered}
f_{y, 1}(t)=f(y)+t, \\
f_{y, 2}(t)=f\left(f_{y, 1}(t)\right)+t, \\
\vdots \\
f_{y, n}(t)=f\left(f_{y, n-1}(t)\right)+t .
\end{gathered}
$$

Then $A_{y}^{\prime}=\left\{t \mid f_{y, n}(t) \in[0, b]\right.$ for some $\left.n \geq 1\right\}$. Let us write

$$
B_{n}(y)=\left\{t \mid f_{y, n}(t) \in[0, b] \text { but } f_{y, m}(t) \notin[0, b] \text { for } m<n\right\} \text {. }
$$

Now $f_{y, n}(t)$ is a map of degree $n$. Hence $B_{n}$ consists of at most $n$ intervals each of length not more than $b /\left(\lambda^{n}+\lambda^{n-1}+\cdots+\lambda+1\right)$. Furthermore, since $f_{y, n}(t)$ changes continuously with $y$, the length of each of these intervals changes continuously with $y$. Hence $m\left(B_{n}(y)\right)$ is a continuous function of $y$, and

$$
m\left(B_{n}(y)\right) \leq m b /\left(\lambda^{n}+\cdots+\lambda+1\right) \quad \text { for all } y .
$$

But $A_{y}^{\prime}=\bigcup_{n=1}^{\infty} B_{n}(y)$ and so $m\left(\bigcup_{j=1}^{n} B_{j}(y)\right)$ converges uniformly to $m\left(A_{y}^{\prime}\right)$. Hence $\boldsymbol{m}\left(A_{y}^{\prime}\right)$ is continuous as required.

From (2.4) it follows by Fubini's Theorem (see e.g. [12, p. 143]) that $m\left(A_{y}\right)=1$ for almost all $y$. But for $y \notin[0, b], m\left(A_{y}\right)=m\left(A_{y}^{\prime}\right)$. Hence by (2.6) it follows that $m\left(A_{b}^{\prime}\right)=1$. Since $A_{b}^{\prime}=[0,1]-E$, as noted above, we have shown $m(E)=0$. To complete the proof we make use of:

(2.7) Proposition (Besicovitch and Taylor [2]). Let $\left(a_{n}\right)_{n=1}^{\infty}$ be a sequence of positive numbers with $\sum_{n=1}^{\infty} a_{n}=1$. Let $E \subset[0,1]$ be a set whose complement is a union of 
intervals $A_{n}$ with $m\left(A_{n}\right)=a_{n}$. Then

$$
\operatorname{dim}_{\mathscr{H}}(E) \leq \inf \left\{\beta \mid \sum_{n=1}^{\infty} a_{n}^{\beta}<\infty\right\} .
$$

Here $\operatorname{dim}_{\mathscr{H}}(E)$ denotes the Hausdorff dimension of $E$. (See [7] for the definition.) For any positive integer $n$ let $\phi(n)$ be the number of positive integers coprime to $n$ and less than $n$. Then as in the proof of (2.6) we see that the complement of $E$, $A_{b}^{\prime}$, consists of $\phi(n)$ intervals for each $n$, each of length not more than

Now

$$
b /\left(\lambda^{n}+\cdots+\lambda+1\right)=\frac{b(\lambda-1)}{\left(\lambda^{n+1}-1\right)} \text {. }
$$

$$
(\lambda-1) \sum_{n=1}^{\infty} \frac{\phi(n)}{\left(\lambda^{n+1}-1\right)^{\beta}}<\infty \quad \text { for all } \beta>0 .
$$

Hence by (2.7) we have $\operatorname{dim}_{\mathscr{H}}(E)=0$. The proof of theorem $\mathrm{I}^{\prime}$ is complete.

(2.8) Example. Consider the 2-parameter family of endomorphisms of the circle defined by

$$
f_{a, t}(x)= \begin{cases}t & \text { if } x \leq a, \\ \frac{x-a}{1-a}+t & \text { if } x \geq a,\end{cases}
$$

for $x \in S^{1}, t \in[0,1]$ and $0<a<1$. (See figure 4.) For any fixed $a, f_{a, 0}$ satisfies the hypothesis of theorem $\mathrm{l}^{\prime}$. In the same way as Arnold and Herman do for diffeomorphisms ([1, p. 273], [5, p. 280]) we can consider level sets for the rotation number.

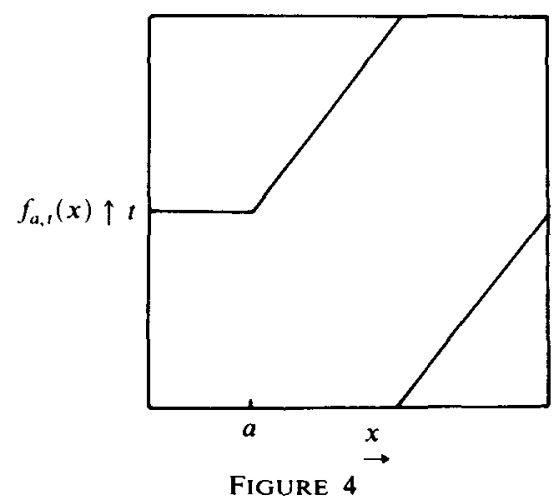

To each rational number a 'balloon' is attached, being the level set for that rotation number. (These balloons are analogous to the so-called 'Arnold tongues'.) Even though the width of each balloon tends to zero as a does, theorem $l^{\prime}$ tells us that for each $a_{0}>0$, the line at height $a_{0}$ intersects the balloons in a set of measure one (see figure 5).

\section{Proof of theorem 2}

We turn to the proof of theorem 2. From now on we will write $f_{Y}$ for the Poincare map of a vector field $Y \in \mathcal{N}$, and $\rho\left(f_{Y}\right)$ for its rotation number. As in [10], the 


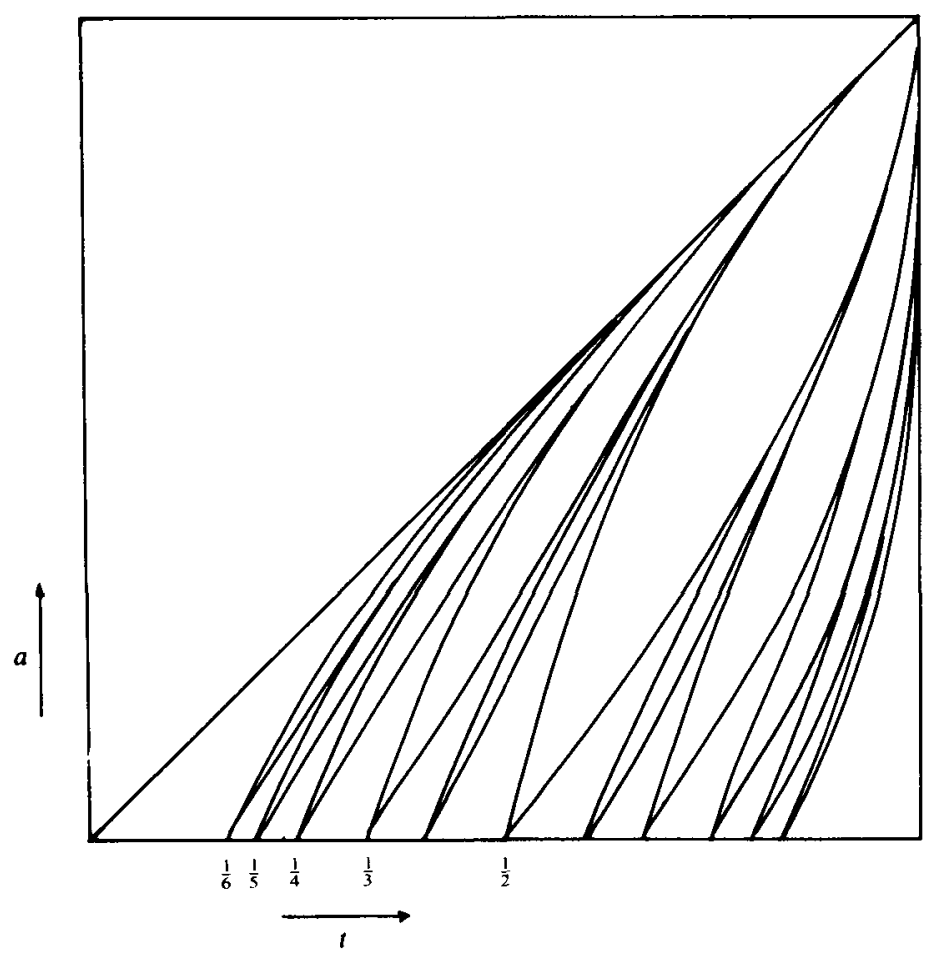

FIGURE 5

procedure is to construct, for each irrational $\alpha \in[0,1)$, a $C^{1}$ function $g_{\alpha}: \mathcal{N} \rightarrow \mathbb{R}$ such that $g_{\alpha}^{-1}(0)=\left\{Y \in \mathcal{N} \mid \rho\left(f_{Y}\right)=\alpha\right\}$ and $D g_{\alpha}(Y) \neq 0$. (Strictly, we will choose $g_{\alpha}$ to have image $S^{1}$.) We first do this for rational rotation numbers $m / n$, using the Implicit Function Theorem to construct $g_{n}, h_{n}$ (for notational convenience we suppress the $m$ 's) which take respectively the lower and upper boundaries of the Morse-Smale class $\left\{Y \mid \rho\left(f_{Y}\right)=m / n\right\}$ onto zero (see figure 6). Then $g_{\alpha}$ is shown to be the $C^{\prime}$ limit of $g_{n_{i}}$ or $h_{n_{j}}$ when $\left(m_{j} / n_{j}\right) \rightarrow \alpha$. More precisely we show that for any $Y \in \mathcal{N}$ and a

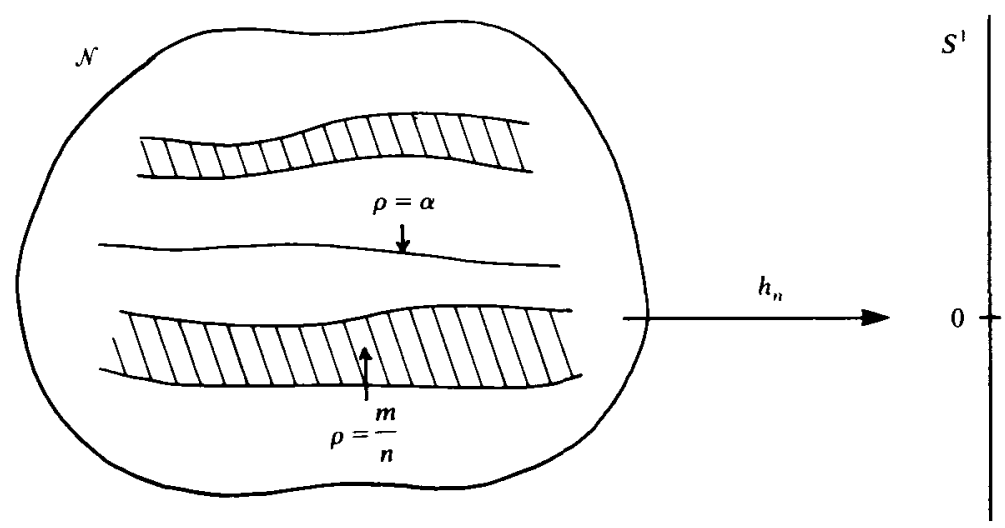

FIGURE 6 
Cauchy sequence $\left(m_{j} / n_{j}\right)_{j=1}^{\infty}$, the sequence $\left(D g_{n_{j}}(Z)\right)_{j=1}^{\infty}$ is uniformly Cauchy for $Z$ in a neighbourhood of $Y$. This also shows that any two of these manifolds that are close are in fact $C^{1}$-close, which is crucial for theorem 3. It turns out that $g_{\alpha}(Y)$ will be the solution for $t$ of $\rho\left(f_{Y}+t\right)=\alpha$; that is how far the graph of $f_{Y}$ must be lifted to have rotation number $\alpha$.

Let $\left[a_{Y}, b_{Y}\right]$ be the interval on which $f_{Y}$ is constant, and let $f(y)=t_{Y}$ for all $y \in\left[a_{Y}, b_{Y}\right]$. We may consider $a, b$ and $t$ to be functions of $Y, \mathcal{N} \rightarrow S^{1}$ and by the Stable Manifold Theorem (see e.g. [6]) they are of class $C^{\infty}$ (consider figure 7). We

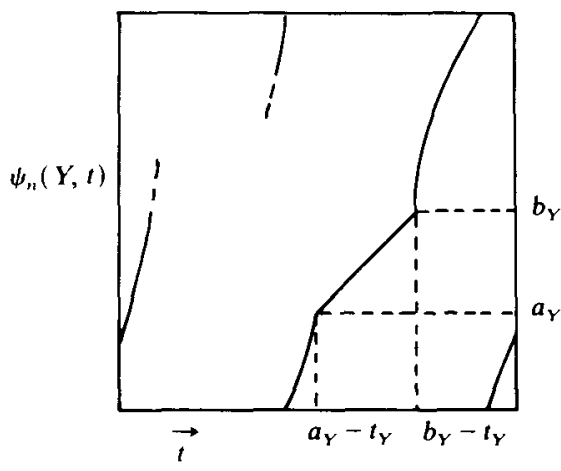

FIGURE 7

will write $f_{Y}+t$ for the function defined by $\left(f_{Y}+t\right)(x)=f_{Y}(x)+t$. The $f_{Y}+t$ has a point of period $n$ exactly when $\left(f_{Y}+t\right)^{n-1}\left(t_{Y}+t\right) \in\left[a_{Y}, b_{Y}\right]$. Define $\psi_{n}: \mathcal{N} \times S^{1} \rightarrow S^{1}$ by

$$
\psi_{n}(Y, t)=\left(f_{Y}+t\right)^{n-1}\left(t_{Y}+t\right) .
$$

So $\psi_{n}(Y, \cdot)$ is a circle map of degree $n$. The boundary points of the Morse-Smale class with rotation number $m / n$ are given by the $m$ th solutions of $\psi_{n}(Y, t)=a_{Y}$ or $b_{Y}$ where the solutions are counted with increasing $t, a_{Y}-t_{Y}$ and $b_{Y}-t_{Y}$ being the zeroth.

Now in a neighbourhood of such a solution $\left(Y, t_{0}\right), \psi_{n}$ is of class $C^{\infty}$. This follows since outside $\left[a_{Y}, b_{Y}\right], f_{Y}$ is a 'genuine' Poincaré map and so the map $(Y, t) \mapsto f_{Y}(t)$ is of class $C^{\infty}$ (see $\left[10\right.$, p. 9]). Also it is clear that $D_{2} \psi_{n}\left(Y, t_{0}\right)>0$. Hence we may apply the Implicit Function Theorem $([4$, p. 270]). This tells us that there is a neighbourhood $\mathscr{B}$ of $Y$ in $\mathcal{N}$ and $C^{\infty}$ functions $g_{n}, h_{n}: \mathscr{B} \rightarrow S^{1}$ satisfying

$$
\begin{gathered}
\psi_{n}\left(Y, g_{n}(Y)\right)-a(Y)=0 \\
\psi_{n}\left(Y, h_{n}(Y)\right)-b(Y)=0
\end{gathered}
$$

and hence

$$
D g_{n}(Y)=-\frac{D_{1} \psi_{n}\left(Y, g_{n}(Y)\right)-D a(Y)}{D_{2} \psi_{n}\left(Y, g_{n}(Y)\right)}
$$

We may write it in this form since $D_{2} \psi_{n}($, ) is a real number. Obviously we have the same formula for $D h_{n}$ with $D a(Y)$ replaced by $D b(Y)$. 
Thus $g_{n}(Y)-a(Y)$ is a $C^{\infty}$ function of $Y$ taking the saddle connection fields with rotation number $m / n$ to zero in $S^{1}$. Note that $D\left(g_{n}(Y)-a(Y)\right) \neq 0$, since, for example, it is non-zero on our particular path $\phi$ in $\S 2$. This shows that these vector fields with saddle connections form a $C^{\infty}$ codimension one submanifold of $\mathcal{N}$, as is known from [10]. Note also that $g_{n}(Y)-a(Y)$ is defined for all $Y \in \mathcal{N}$ and $C^{\infty}$ everywhere.

From now on fix $Y \in \mathcal{N}$ and choose a Cauchy sequence of rationals $\left\{m_{j} / n_{j}\right\}_{j=1}^{\infty}$. To prove theorem 2 it is sufficient to show that $\left\{D g_{n_{j}}(Z)\right\}_{j=1}^{\infty}$ is uniformly Cauchy for $Z$ in a neighbourhood of $Y$ as $j \rightarrow \infty$ (see [4, p. 163]). It will be clear from the proof that we could have allowed any Cauchy sequence of saddle connection fields and their corresponding functions $h_{n_{j}}$ or $g_{n_{j}}$. For simplicity we just consider the functions $g_{n_{j}}$.

We may write $f_{Y}$ as a map of two variables: $f(Y, t)=f_{Y}(t)$. We rewrite $D g_{n}$ in terms of $D_{1} f$ and $D_{2} f$. For $n \geq 2$, let $\mu(Y, t, s)=f_{Y}(t)+s$ and $\nu(Y, t)=$ $\left(Y, \psi_{n-1}(Y, t), t\right)$. Then $\psi_{n}=\mu \circ \nu$. Hence, by the chain rule,

$$
\begin{gathered}
D \psi_{n}(Y, t)=\left(D_{1} f\left(\psi_{n-1}(Y, t)\right)+D_{2} f\left(\psi_{n-1}(Y, t)\right) \cdot D_{1} \psi_{n-1}(Y, t),\right. \\
\left.D_{2} f\left(\psi_{n-1}(Y, t)\right) \cdot D_{2} \psi_{n-1}(Y, t)+1\right) .
\end{gathered}
$$

Hence from (3.1) and by induction

$$
D g_{n}(Y)=-\frac{\alpha_{n, n}+\beta_{n, n}\left(\alpha_{n, n-1}+\beta_{n, n-1}\left(\alpha_{n, n-2} \cdots\left(\alpha_{n, 2}+\beta_{n, 2} \cdot D t(Y)\right) \cdots\right)-D a(Y)\right.}{\beta_{n, n}\left(\beta_{n, n-1}\left(\cdots\left(\beta_{n, 2}+1\right)+1\right) \cdots\right)+1}
$$

where $\quad \alpha_{n, j}=D_{1} f\left(Y, x_{j-1}\right) \quad$ and $\quad \beta_{n, j}=D_{2} f\left(Y, x_{j-1}\right)$ and here $x_{j}=$ $\left(f_{Y}+g_{n}(Y)\right)^{j-1}\left(t_{Y}+g_{n}(Y)\right)$ - that is the $(j-1)$ st iterate of $t_{Y}+g_{n}(Y)$ which is in the periodic orbit for $f_{Y}+g_{n}(Y)$. Since the $\beta_{n, j}$ 's are real numbers, (3.2) is the same as

$$
\begin{aligned}
D g_{n}(Y)= & \frac{-\alpha_{n, n}}{\beta_{n, n}\left(\beta_{n, n-1}(\cdots)+1\right)+1}+\frac{-\alpha_{n, n-1}}{\beta_{n, n-1}(\cdots)+1+1 / \beta_{n, n}}+\cdots \\
& \cdots+\frac{-D t(Y)}{1+\frac{1}{\beta_{n, 2}}+\cdots+\frac{1}{\beta_{n, 2} \cdots \beta_{n, n}}} \\
& +\frac{D a(Y)}{\beta_{n, n}\left(\beta_{n, n-1}(\cdots)+1\right)+1} .
\end{aligned}
$$

So to calculate $D g_{n}$ we need to know the values of $D_{1} f\left(Y, x_{j}\right)$ and $D_{2} f\left(Y, x_{j}\right)$ for $x_{j}$ in the periodic orbit of $f_{Y}+g_{n}(Y)$. To deal with the case when this orbit comes close to $a_{Y}$ or $b_{Y}$ we need:

(3.4) Proposition.

$$
\begin{array}{ll}
-\frac{D_{1} f\left(Z, a_{Z}-\delta\right)}{D_{2} f\left(Z, a_{Z}-\delta\right)} \rightarrow D a(Z) & \text { as } \delta \rightarrow 0^{+} \\
-\frac{D_{1} f\left(Z, b_{Z}+\delta\right)}{D_{2} f\left(Z, b_{Z}+\delta\right)} \rightarrow D b(Z) & \text { as } \delta \rightarrow 0^{+}
\end{array}
$$


(3.5) Corollary. $\left\|D_{1} f(Z, x) / D_{2} f(Z, x)\right\|$ is uniformly bounded for $x \notin\left[a_{Z}, b_{Z}\right]$ and $Z$ in a small neighbourhood of $Y$.

Since the proof of (3.4) is quite technical, we defer it to the next section.

Fix $\gamma>0$. We will find $\eta$ so small that for $Z$ in a small neighbourhood of $Y$,

$$
\left|g_{s}(Z)-g_{\bar{s}}(Z)\right|<\eta \Rightarrow\left\|D g_{s}(Z)-D g_{\bar{s}}(Z)\right\|<\gamma,
$$

where $r / s$ and $\bar{r} / \bar{s}$ are elements of $\left\{m_{j} / n_{j}\right\}_{j=1}^{\infty}$. The first step is to show that we can ignore all but finitely many terms in (3.3). We write its $j$ th term

$$
T_{n}^{j}=\frac{-\alpha_{n, j}}{\beta_{n, j}\left(\beta_{n, j-1}(\cdots)+1\right)+1+\frac{1}{\beta_{n, j+1}}+\cdots+\frac{1}{\beta_{n, j+1} \cdots \beta_{n, n}}} .
$$

This is defined for $1 \leq j \leq n$ by letting $\alpha_{n, 1}=D t(Y)$. Then if $M$ is the uniform bound on $\left\|D_{1} f(Z, x) / D_{2} f(Z, x)\right\|$ for $Z$ in a neighbourhood $A$ of $Y$, guaranteed by (3.5) we have $\left\|T_{n}^{j}\right\| \leq M / \lambda^{j-2}$. So we may choose $n_{0}$ so large that

$$
\sum_{j=n_{0}}^{\infty} \frac{M}{\lambda^{j-2}}<\frac{\gamma}{8}
$$

and also

$$
\frac{\|D a(Z)\|}{\lambda^{n_{0}}}<\frac{\gamma}{8} \text { and } \frac{\|D b(Z)\|}{\lambda^{n_{0}}}<\frac{\gamma}{8},
$$

for all $Z \in A$, if $A$ is small enough. Next we choose $\delta_{1}$ so small that if $x_{j}$ comes within $\delta_{1}$ of $a_{Z}$ or $b_{Z}$ then $\beta_{n, j}$ is large enough so that we may ignore $T_{n}^{i}$ for $i>j$. Precisely, choose $\delta_{1}>0$ so that for all $Z \in A$ and $x \in\left(a_{z}-\delta_{1}, a_{z}\right)$ or $x \in\left(b_{z}, b_{z}+\delta_{1}\right)$ the following hold:

$$
\begin{gathered}
\frac{1}{D_{2} f(Z, x)} \sum_{j=2}^{\infty} \frac{M}{\lambda^{j-2}}<\frac{\gamma}{8} . \\
\frac{\|D a(Z)\|}{D_{2} f(Z, x)}<\frac{\gamma}{8} \text { and } \frac{\|D b(Z)\|}{D_{2} f(Z, x)}<\frac{\gamma}{8} . \\
\left\|\frac{D_{1} f(Z, x)}{D_{2} f(Z, x)}+D a(Z)\right\|<\frac{\gamma}{8} \quad \text { if } x \in\left(a_{Z}-\delta_{1}, a_{Z}\right), \\
\left\|\frac{D_{1} f(Z, x)}{D_{2} f(Z, x)}+D b(Z)\right\|<\frac{\gamma}{8} \quad \text { if } x \in\left(b_{Z}, b_{Z}+\delta_{1}\right) . \\
\frac{\lambda}{(\lambda-1)} \frac{1}{D_{2} f(Z, x)}<\frac{\gamma}{8 \cdot M \cdot n_{0}} .
\end{gathered}
$$

The inequalities (3.10) are possible by using (3.4). Now choose $0<\delta_{2}<\delta_{1}$. Let us write

$$
\begin{aligned}
& x_{j}=\left(f_{Z}+g_{s}(Z)\right)^{j-1}\left(t_{Z}+g_{s}(Z)\right) \\
& y_{j}=\left(f_{Z}+g_{\bar{s}}(Z)\right)^{j-1}\left(t_{Z}+g_{\bar{s}}(Z)\right) .
\end{aligned}
$$

The idea is to choose $\eta$ so small that $\left|g_{s}(Z)-g_{\bar{s}}(Z)\right|<\eta$ implies that if $x_{j}$ is $\delta_{2}$-close to $a_{z}$ or $b_{z}$ then $y_{j}$ is $\delta_{1}$-close to $a_{z}$ or $b_{z}$. We may choose $\eta$ small enough so that 
if $\left|g_{i}(Z)-g_{i}(Z)\right|<\eta$ then the following holds:

if $x_{j}$ and $y_{j}$ are inside $\left[b_{z}+\delta_{2}, a_{z}-\delta_{2}\right]$ then

$$
\left\|T_{s}^{j}-T_{\bar{s}}^{j}\right\| \leq \gamma / 4 n_{0} \quad \text { for } 1 \leq j \leq n_{0} .
$$

Note that for fixed $j$, to make $\left\|T_{s}^{j}-T_{\bar{s}}^{j}\right\|<\gamma / 4 n_{0}$, it is necessary only to make $\beta_{s, k}$ and $\beta_{\bar{s}, k}$ close for finitely many $k$, say $N(j)$. Therefore we may choose $n_{1}$ so that if $\beta_{s, k}$ and $\beta_{\bar{s}, k}$ are close for $1 \leq k \leq n_{1}$ then $\left\|T_{s}^{j}-T_{s}^{j}\right\|<\gamma / 4 n_{0}$ for $0 \leq j \leq n_{0}$, where $n_{1}=\sup \left\{N(j) \mid 1 \leq j \leq n_{0}\right\}$. To take care of the case $s<n_{1}$ we also need

$$
\begin{aligned}
& \text { if } K=\sup _{x \in\left[b_{Z}+\delta_{2}, a_{Z}-\delta_{2}\right], Z \in A}\left|D_{2} f(Z, x)\right| \text { then } \\
& \delta_{2}-\delta_{1}>\eta \cdot K^{n_{2}-1} \quad \text { where } n_{2}=\max \left(n_{0}, n_{1}\right) .
\end{aligned}
$$

This tells us that if $x_{j} \notin\left[b_{Z}+\delta_{2}, a_{Z}-\delta_{2}\right]$ then $y_{j} \notin\left[b_{Z}+\delta_{1}, a_{Z}-\delta_{1}\right]$, for $1 \leq j \leq n_{2}$. So using (3.11), the worst possible case for (3.12) is

$$
\begin{aligned}
\left\|T_{s}^{j}-T_{\bar{s}}^{j}\right\| \leq & \left\|\frac{\alpha_{s, j}}{\beta_{s, j}\left(\beta_{s, j-1}(\cdots)+1\right)+1}-\frac{\alpha_{\bar{s}, j}}{\beta_{\bar{s}, j}\left(\beta_{\bar{s}, j-1}(\cdots)+1\right)+1+\gamma / 8 M n_{0}}\right\| \\
\leq & \left\|\frac{\left(\beta_{\bar{s}, j}\left(\beta_{\bar{s}, j-1}(\cdots)+1\right) \alpha_{s, j}-\left(\beta_{s, j}(\cdots)+1\right) \alpha_{\bar{s}, j}\right)}{\left(\beta_{s, j}(\cdots)+1\right)\left(\beta_{\bar{s}, j}(\cdots)+1+\gamma / 8 M n_{0}\right)}\right\| \\
& +\left\|\frac{\alpha_{s, j} \gamma / 8 M n_{0}}{\left(\beta_{s, j}(\cdots)+1\right)\left(\beta_{\bar{s}, j}(\cdots)+1+\gamma / 8 M n_{0}\right.}\right\| \\
\leq & \gamma / 4 n_{0}
\end{aligned}
$$

if $\beta_{s, k}$ and $\beta_{\tilde{s}, k}$ are close for $1 \leq k \leq j$ and $\alpha_{s, j}$ and $\alpha_{\bar{s}, j}$ are close, which is true if $\left|g_{s}(Z)-g_{\bar{s}}(Z)\right|$ is small enough.

In a similar way we can ensure that if $x_{j}$ and $y_{j}$ are both in $\left(a_{Z}-\delta_{1}, a_{z}\right]$ or $\left[b_{Z}, b_{Z}+\delta_{1}\right)$ then for $\left|g_{s}(Z)-g_{\bar{s}}(Z)\right|<\eta$ the following holds:

$$
\begin{aligned}
\|D a(Z)\| & \left(\frac{1}{\beta_{s, j}(\cdots)+1+\left(1 / \beta_{s, j+1}\right)+\cdots+\left(1 / \beta_{s, j+1} \cdots \beta_{s, s}\right)}\right. \\
& \left.-\frac{1}{\beta_{\bar{s}, j}(\cdots)+1+\left(1 / \beta_{\bar{s}, j+1}\right)+\cdots+\left(1 / \beta_{\bar{s}, j+1} \cdots \beta_{\bar{s}, \bar{s}}\right)}\right)<\frac{\gamma}{8},
\end{aligned}
$$

for $1 \leq j \leq n_{0}$ and $Z \in A$. We now claim that

$$
\left|g_{s}(Z)-g_{s}(Z)\right|<\eta \Rightarrow\left\|D g_{s}(Z)-D g_{s}(Z)\right\|<\gamma .
$$

We consider three cases:

Case 1. $x_{j}$ and $y_{j}$ are in $\left[b_{z}+\delta_{2}, a_{Z}-\delta_{2}\right]$ for $1 \leq j \leq n_{0}$. Consider the equation (3.3). The terms $T_{s}^{j}, T_{\bar{s}}^{j}$ are taken care of, for $j>n_{0}$, by (3.6), and each final term by (3.7). The other terms are dealt with by (3.12). Thus

$$
\left\|D g_{s}(Z)-D g_{\vec{s}}(Z)\right\|<\frac{\gamma}{4}+\frac{\gamma}{8}+\frac{\gamma}{8}+\frac{\gamma}{8}+\frac{\gamma}{8}<\gamma
$$

If case 1 does not happen let $j_{0}$ be the first $j$ where it fails. Suppose $x_{j_{0}} \notin\left[b_{Z}+\delta_{2}, a_{Z}-\delta_{2}\right]$. Clearly the case $y_{j_{0}} \notin\left[b_{Z}+\delta_{2}, a_{Z}-\delta_{2}\right]$ is similar.

Case 2. $x_{j_{0}}=a_{z}$. This is the case $s=j_{0}$, since there is a point of period $j_{0}$. If also $y_{j_{b}}=a_{Z}$ then $D g_{s}$ and $D g_{s}$ differ only in their final terms and this case is clearly all right. Otherwise, by (3.13), $y_{j_{0}} \in\left(a_{Z}-\delta_{1}, a_{z}\right)$. For $j \leq j_{0}$ the terms $T_{s}^{j}, T_{s}^{j}$ are dealt 
with by (3.12) and for $j \geq j_{0}+1, T_{\bar{s}}^{j}$ are dealt with by (3.8). Hence

$$
\begin{gathered}
\left\|D g_{s}(Z)-D g_{\bar{s}}(Z)\right\|<\frac{j_{0} \cdot \gamma}{4 n_{0}}+\frac{\gamma}{8}+\left\|\frac{D a(Z)}{\beta_{s, s}\left(\beta_{s, s-1}(\cdots)+1\right)+1}-T_{\bar{s}}^{j_{j}+1}\right\| \\
+\frac{\|D a(Z)\|}{\beta_{\overline{s, s}}\left(\beta_{\bar{s}, \bar{s}-1}(\cdots)+1\right)+1}
\end{gathered}
$$

Since $y_{j_{0}} \in\left(a_{Z}-\delta_{1}, a_{Z}\right)$ it follows by (3.10) that

$$
\left\|\frac{D_{1} f\left(Z, y_{j_{0}}\right)}{D_{2} f\left(Z, y_{j_{0}}\right)}+D a(z)\right\|<\frac{\gamma}{8} \quad \text { or } \quad\left\|\frac{\alpha_{\bar{s}, j_{0}+1}}{\beta_{\bar{s}, j_{0}+1}}+D a(Z)\right\|<\frac{\gamma}{8} .
$$

Thus

$$
\left\|T_{\bar{s}}^{j_{0}+1}-\frac{\operatorname{Da}(Z)}{\beta_{\bar{s}, j_{0}}(\cdots)+1+\cdots+\left(1 / \beta_{\bar{s}, j_{0}+1} \cdots \beta_{\bar{s}, \bar{s}}\right)}\right\|<\frac{\gamma}{8},
$$

and so

$$
\begin{aligned}
& \left\|\frac{D a(Z)}{\beta_{s, s}\left(\beta_{s, s-1}(\cdots)+1\right)+1}-T_{\bar{s}}^{j_{0}+1}\right\| \\
& \quad \leq \frac{\gamma}{8}+\left\|\frac{D a(Z)}{\beta_{s, s}\left(\beta_{s, s-1}(\cdots)+1\right)+1}-\frac{D a(Z)}{\beta_{\bar{s}, j_{0}}(\cdots)+1+\cdots+\left(1 / \beta_{\bar{s}, j_{0}+1} \cdots \beta_{\bar{s}, \bar{s}}\right)}\right\| \\
& \quad<\frac{\gamma}{8}+\frac{\gamma}{8} \quad \text { by }(3.14) .
\end{aligned}
$$

Finally, by (3.7)

$$
\frac{\|D a(Z)\|}{\beta_{\bar{s}, \bar{s}}\left(\beta_{\bar{s}, \bar{s}-1}(\cdots)+1\right)+1}<\frac{\gamma}{8}
$$

Thus

$$
\left\|D g_{s}(Z)-D g_{\bar{s}}(Z)\right\|<\frac{\gamma}{4}+\frac{\gamma}{8}+\frac{\gamma}{8}+\frac{\gamma}{8}<\gamma
$$

Case 3. $x_{j_{0}} \in\left(a_{z}-\delta_{2}, a_{z}\right)$. Clearly the case $x_{j_{0}} \in\left(b_{z}, b_{z}+\delta_{2}\right)$ is similar. By (3.13), $y_{j_{0}} \in\left(a_{Z}-\delta_{1}, a_{Z}\right)$. Using (3.12) and (3.8) as in case 2 , we have

$$
\begin{aligned}
\left\|D g_{s}(Z)-D g_{\bar{s}}(Z)\right\|< & \frac{j_{0} \cdot \gamma}{4 n_{0}}+\frac{\gamma}{8}+\frac{\gamma}{8} \\
& +\| \frac{\alpha_{s, j_{0}+1}}{\beta_{s, j_{0}+1}(\cdots)+1+\cdots+\left(1 / \beta_{s, j_{0}} \cdots \beta_{s, s}\right)} \\
& -\frac{\alpha_{\bar{s}, j_{0}+1}}{\beta_{\overline{s, j} j_{0}+1}(\cdots)+1+\cdots+\left(1 / \beta_{\bar{s}, j_{0}} \cdots \beta_{\bar{s}, \bar{s}}\right)} \| \\
& +\|\operatorname{Da}(Z)\|\left(\frac{1}{\beta_{s, s}(\cdots)+1}-\frac{1}{\beta_{\bar{s}, \bar{s}}(\cdots)+1}\right) .
\end{aligned}
$$


Since both $x_{j_{0}}$ and $y_{j_{0}}$ are in $\left(a_{z}-\delta_{1}, a_{z}\right)$ it follows from (3.10) that

$$
\begin{aligned}
& \| \frac{\alpha_{s, j_{0}+1}}{\beta_{s, j_{0}+1}(\cdots)+1+\cdots+\left(1 / \beta_{s, j_{0}+2} \cdots \beta_{s, s}\right)} \\
& \quad-\frac{\alpha_{\bar{s}, j_{0}+1}}{\beta_{\bar{s}, j_{0}+1}(\cdots)+1+\cdots+\left(1 / \beta_{\bar{s}, j_{0}+2} \cdots \beta_{\bar{s}, \bar{s}}\right)} \| \\
& <\frac{\gamma}{8}+\frac{\gamma}{8}+\|D a(Z)\|\left(\frac{1}{\beta_{s, j_{0}}(\cdots)+1+\cdots+\left(1 / \beta_{s, j_{0}+1} \cdots \beta_{s, s}\right)}\right. \\
& \left.\quad-\frac{1}{\beta_{\bar{s}, j_{0}}(\cdots)+1+\cdots+\left(1 / \beta_{\bar{s}, j_{0}+1} \cdots \beta_{\bar{s}, \bar{s}}\right)}\right) \\
& <\frac{\gamma}{4}+\frac{\gamma}{8} \quad \text { by (3.14). }
\end{aligned}
$$

By applying (3.7) to the final term we now have

$$
\left\|D g_{s}(Z)-D g_{\bar{s}}(Z)\right\|<\frac{\gamma}{2}+\frac{\gamma}{4}+\frac{\gamma}{8}+\frac{\gamma}{8}=\gamma
$$

Thus theorem 2 is proved.

4. Proof of (3.4) and (3.5)

The idea of the proof of (3.4) is to show that near to the saddle separatrix $f(Z, x)$ behaves like the Poincaré map of a linear saddle. But for a linear vector field $L$ with matrix

$$
\left(\begin{array}{cc}
\lambda(L) & 0 \\
0 & -\mu(L)
\end{array}\right)
$$

its Poincaré map $p(L, x)$ behaves like $x \mapsto x^{\mu(L) / \lambda(L)}$. Hence $D_{1} p(L, x) / D_{2} p(L, x)$ behaves like

$$
\frac{D(\mu(L) / \lambda(L)) \cdot x^{\mu(L) / \lambda(L)} \log x}{x^{(\mu(L) / \lambda(L))-1}}=D(\mu(L) / \lambda(L)) \cdot x \log x \rightarrow 0
$$

as $x \rightarrow 0$. Because the saddle point of $Z$ moves with $Z$ the term $D a(Z)$ or $D b(Z)$ enters as a correction term. Let us call the saddle point of $Z, S(Z)$. By Sell's Linearization Theorem [9] there is a $C^{1}$ map $l_{Z}: U \rightarrow \mathbb{B}^{2}$ from some neighbourhood $U$ of $S(Z)$ conjugating the flow to its linear part $L_{Z}=D Z_{S(Z)}$ :

$$
l_{Z}\left(\psi_{Z}(x, t)\right)=\psi_{L_{Z}}\left(l_{Z}(x), t\right)
$$

if $x$ and $\psi_{Z}(x, t) \in U$. (Here $\psi($,$) denotes the flow induced by the vector field$ $X$.) Furthermore, Sell shows that the linearization can be chosen to depend in a $C^{1}$ way upon $Z([9, \mathrm{p} .64])$, so $l(Z, t)$ is a $C^{\prime}$ map. Therefore we choose $l(Z, t)$ first for $Z=Y$. Then the neighbourhood referred to in (3.5) in which $Z$ is allowed to lie is the domain of definition of the first component of $l$. Call this neighbourhood $\mathscr{B}$. For points inside $\bigcup_{t \in R} \psi_{Z}(t, U)$ we can extend $l_{Z}$, so long as its domain of definition does not overlap itself, by setting

$$
l_{Z}(x)=\psi_{L_{Z}}\left(l_{Z}\left(\psi_{Z}(x, t),-t\right)\right.
$$

where $t$ is chosen so that $\psi_{Z}(x, t) \in U$ and so that the partial orbit joining $x$ to $\psi_{Z}(x, t)$ has not passed through $U$. Hence we may extend the domain of $l_{Z}$ so far 

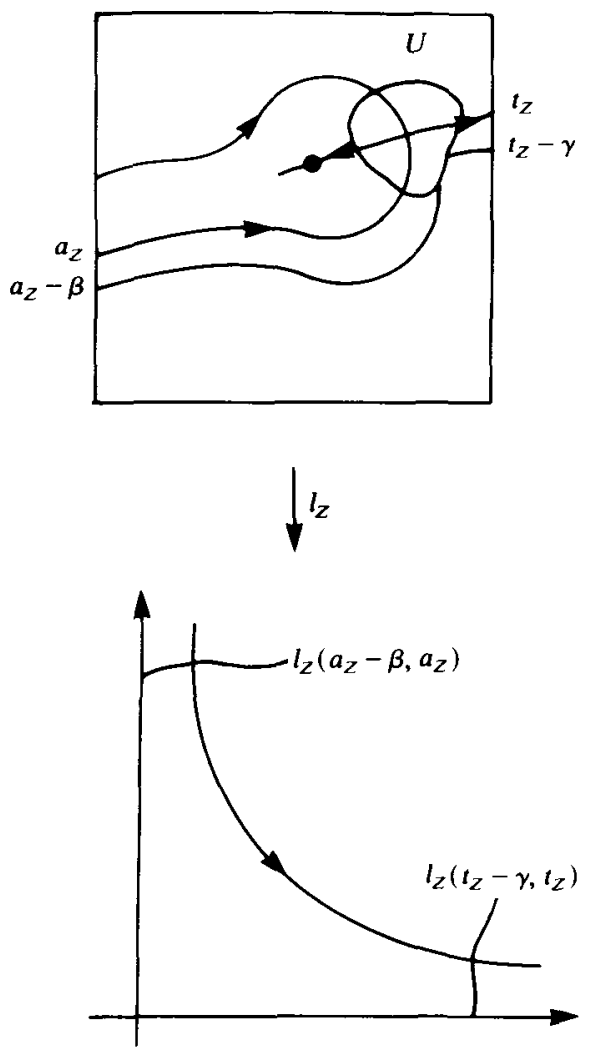

Figure 8a

as to include intervals in $\Sigma,\left(a_{Z}-\beta, a_{Z}\right)$ and $\left(t_{Z}-\gamma, t_{Z}\right)$ for small $\beta, \gamma$. (See figure 8a.) Note that $l(Z, t)$ is still a $C^{1}$ map on this extended domain. Now for $t \in\left(a_{Z}-\beta, a_{Z}\right)$ let

$$
l(Z, t)=\left(\begin{array}{c}
x(Z, t) \\
\sigma(x(Z, t))
\end{array}\right)
$$

and for $t \in\left(t_{Z}-\gamma, t_{Z}\right)$ let

$$
l(Z, t)=\left(\begin{array}{c}
\tau(y(Z, t)) \\
y(Z, t)
\end{array}\right),
$$

where $\sigma:(0, \bar{\beta}) \rightarrow \mathbb{R}$ and $\tau:(0, \bar{\gamma}) \rightarrow \mathbb{R}$ are $C^{1}$ functions for some small $\bar{\beta}$ and $\bar{\gamma}$. (See figure $8 \mathrm{~b}$.) So for $t \in\left(a_{Z}-\beta, a_{Z}\right), f_{Z}(t)$ progresses thus:

$$
t \longmapsto t_{Z}\left(\begin{array}{c}
x(Z, t) \\
\sigma(x(Z, t))
\end{array}\right) \underset{p_{Z}}{\longmapsto}\left(\begin{array}{c}
\tau_{Z}\left(p_{Z}(x(Z, t))\right) \\
p_{Z}(x(Z, t))
\end{array}\right) \underset{t_{Z}^{-1}}{\longmapsto} f_{Z}(t)
$$

where $p_{Z}:[0, \bar{\beta}) \rightarrow[0, \bar{\gamma})$ is the Poincaré map of $L_{Z}$ from $l_{Z}\left(a_{Z}-\beta, 0\right)$ to $l_{Z}\left(t_{Z}-\gamma, 0\right)$.

Let $\Pi_{x}: l_{z}\left(a_{z}-\beta, a_{z}\right] \rightarrow \mathbb{R}$ and $\Pi_{y}: l_{z}\left(t_{z}-\gamma, t_{z}\right) \rightarrow \mathbb{R}$ be the projections:

$$
\Pi_{x}\left(\begin{array}{c}
x \\
\sigma_{Z}(x)
\end{array}\right)=x \text { and } \Pi_{y}\left(\begin{array}{c}
\tau_{Z}(y) \\
y
\end{array}\right)=y .
$$




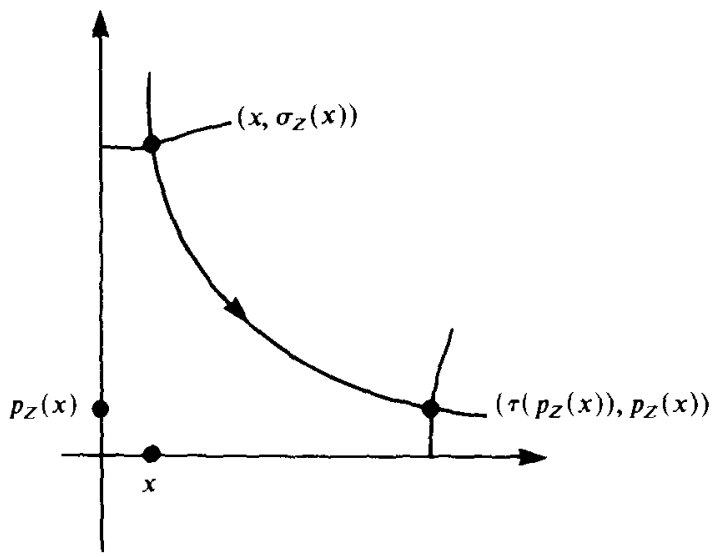

FIGURE $8 b$

Then if we set

$$
\begin{aligned}
& j(Z, t)=\Pi_{x} \circ l(Z, t) \\
& k(Z, t)=l_{Z}^{-1} \circ \Pi_{y}^{-1}(Z, t)
\end{aligned}
$$

we have

$$
f(Z, t)=k\left(Z, p_{Z}(j(Z, t))\right) .
$$

Let us write $F_{\delta}(Z)=f\left(Z, a_{Z}-\delta\right)$. Then

$$
D F_{\delta}(Z)=D_{1} f\left(Z, a_{Z}-\delta\right)+D_{2} f\left(Z, a_{Z}-\delta\right) \cdot D a(Z) \text {. }
$$

On the other hand we also have

$$
F_{\delta}(Z)=k\left(Z, p_{Z}\left(j\left(Z, a_{Z}-\delta\right)\right)\right)
$$

and so

(4.3) $\quad D F_{\delta}(Z)=D_{1} k+D_{2} k \cdot D_{1} p+D_{2} k \cdot D_{2} p \cdot D_{1} j+D_{2} k \cdot D_{2} p \cdot D_{2} j \cdot D a(Z)$.

(In order to simplify the equations, from now on we are omitting the points at which $D k, D j$ and $D p$ are evaluated.) From (4.1) we also have

$$
D_{2} f(Z, t)=D_{2} k \cdot D_{2} p \cdot D_{2} j .
$$

Together (4.2), (4.3) and (4.4) give us

$$
\frac{D_{1} f\left(Z, a_{Z}-\delta\right)}{D_{2} f\left(Z, a_{Z}-\delta\right)}=\frac{D_{1} k}{D_{2} k \cdot D_{2} p \cdot D_{2} j}+\frac{D_{1} p}{D_{2} p \cdot D_{2} j}+\frac{D_{1} j}{D_{2} j} .
$$

We consider each term on the right-hand side separately.

$$
\begin{aligned}
\lim _{\delta \rightarrow 0} \frac{D_{1} k}{D_{2} k \cdot D_{2} p \cdot D_{2} j} & =\frac{D_{1} k(Z, 0)}{D_{2} k(Z, 0) D_{2} j(Z, 0)} \cdot \lim _{\delta \rightarrow 0} \frac{1}{D_{2} p} \\
& =0,
\end{aligned}
$$

as we will see below. Note that this convergence is uniform in $\mathscr{B}$ since $j$ and $k$ are bounded in $\mathscr{B}$.

$$
\lim _{\delta \rightarrow 0} \frac{D_{1} p\left(Z, j\left(Z, a_{Z}-\delta\right)\right)}{D_{2} p\left(Z, j\left(Z, a_{Z}-\delta\right)\right)}=\lim _{x \rightarrow 0} \frac{D_{1} p(Z, x)}{D_{2} p(Z, x)} \cdot \frac{1}{D_{2} j\left(Z, a_{Z}\right)} .
$$


We consider this case below. For the first term let us write $J(Z, \delta)=j\left(Z, a_{Z}-\delta\right)$. Then

$$
\frac{D_{1} J(Z, \delta)}{D_{2} J\left(Z, a_{Z}-\delta\right)}=\frac{D_{1} j}{D_{2} j}+D a(Z) .
$$

Since $D_{1} J(Z, 0)=0$ and $J$ is $C^{1}$, we deduce $\lim _{\delta \rightarrow 0} D_{1} j / D_{2} j=-D a(Z)$. Thus from (4.5) we now have

$$
\lim _{\delta \rightarrow 0} \frac{D_{1} f\left(Z, a_{Z}-\delta\right)}{D_{2} f\left(Z, a_{Z}-\delta\right)}=-D a(Z)+\frac{1}{D_{2} j\left(Z, a_{Z}\right)} \lim _{x \rightarrow 0} \frac{D_{1} p(Z, x)}{D_{2} p(Z, x)} .
$$

Hence it now suffices to examine $p(Z, x)$, the Poincaré map of $L_{Z}$. We may assume $L_{Z}$ has the form

$$
\left(\begin{array}{cc}
\lambda(Z) & 0 \\
0 & -\mu(Z)
\end{array}\right)
$$

Let us write $\alpha(Z)=\mu(Z) / \lambda(Z)$. Then we may integrate the vector field $L_{Z}$ to find

$$
p(Z, x)=\sigma_{Z}(x)\left(\frac{x}{\tau_{Z}\left(p_{Z}(x)\right)}\right)^{\alpha(Z)} .
$$

Differentiating we get

$$
\begin{aligned}
& D_{1} p(Z, x)=x^{\alpha(Z)}\left[\frac{D_{1} \sigma \cdot \tau+\sigma \cdot D \alpha[\log x-\log \tau] \tau-\sigma \cdot \alpha \cdot D_{1} \tau}{\tau^{\alpha(Z)+1}+D_{2} \tau \cdot \sigma \cdot x^{\alpha(Z)}}\right] \\
& D_{2} p(Z, x)=x^{\alpha(Z)-1}\left[\frac{D_{2} \sigma \cdot \tau \cdot x+\sigma \cdot \tau \cdot \alpha}{\tau^{\alpha(Z)+1}+\sigma \cdot \alpha \cdot x^{\alpha(Z)} \cdot D_{2} \tau}\right]
\end{aligned}
$$

and hence that

$$
\begin{aligned}
\lim _{x \rightarrow 0} \frac{D_{1} p(Z, x)}{D_{2} p(Z, x)} & =\lim _{x \rightarrow 0} \frac{x\left[\left(D_{1} \sigma \cdot \tau+\sigma \cdot D \alpha[\log x-\log \tau] \tau-\sigma \cdot \alpha \cdot D_{1} \tau\right) \cdot \tau^{\alpha+1}\right]}{\tau^{\alpha+1} \cdot \sigma \cdot \tau \cdot \alpha} \\
& =0
\end{aligned}
$$

since $\lim _{x \rightarrow 0} x \log x=0$. Notice that since $\sigma, \tau$ and $\alpha$ are bounded in $\mathscr{B}$ this convergence is uniform in $\mathscr{B}$. By (4.6) the proof of (3.4) is complete.

Proof of (3.5). Consider (4.5). We have already noted that the first two terms on the right hand side are bounded uniformly in $\mathscr{B}$. But the final term is defined and continuous for $\delta=0$ so is also bounded in $\mathscr{B}$. Thus (3.5) is proved.

\section{Proof of theorem 3}

Let $C^{1}(I, \mathfrak{X})$ be the space of $C^{1}$ paths in $\mathfrak{X}^{\infty}\left(T^{2}\right)$ with the $C^{1}$ topology. We recall what it means for a path to be stable (see e.g. [11]).

(5.1) Definition. Two paths $X, Y \in C^{1}(I, \mathfrak{X})$ are (mildly) equivalent if there exists a reparametrising homeomorphism $h: I \rightarrow I$ such that $X(t)$ and $Y(h(t))$ are topologically equivalent vector fields. $X$ and $Y$ are strongly equivalent if in addition the topological equivalence between $X(t)$ and $Y(h(t))$ can be chosen to change continuously with $t$. $X$ is a stable path if there is a neighbourhood $U$ of $X$ in $C^{1}(I, \mathfrak{X})$ such that all paths $Y$ in $U$ are strongly equivalent to $X$.

Before showing that our chosen path $\phi$ is stable we note that all Cherry fields with the same rotation number are topologically equivalent. Similarly all Morse-Smale 
fields in the neighbourhood $\mathcal{N}$ with the same rotation number are topologically equivalent, as are any fields in the corresponding boundaries - those with saddle connections (only we must distinguish between 'lower' and 'upper' saddle connections). The topological equivalence can be constructed in essentially the same way in all cases, as follows.

Choose $X, Y \in \mathcal{N}$ of the same type and with the same rotation number. We first restrict to the transverse circle $\Sigma$. Call the successive inverse intersections of the stable manifolds of the sinks of $X, Y$ with $\Sigma, I_{j}, \tilde{I}_{j}$ respectively. If the fields $X$ and $Y$ are Morse-Smale, $I_{j}$ and $\tilde{I}_{j}$ will have two components for large enough $j$. The restriction of the topological equivalence to $\Sigma$ is defined by taking $I_{j}$ to $\tilde{I}_{j}$ affinely according to the ratio of their lengths (naturally we deal with the two components of $I_{j}$ and $\tilde{I}_{j}$ separately, if necessary). Since the stable manifold of each sink is dense in $\Sigma$ (cf. (1.2)) the map can be extended uniquely to the whole of $\Sigma$. Because $X$ and $Y$ have the same rotation number the $I_{j}$ 's, $\tilde{I}_{j}$ 's intersect $\Sigma$ in the same order, and so the map is indeed continuous. The map may now be extended to the whole of $\Sigma$, but care must be taken near the saddle separatrices.

For points $x, y$ in the same orbit we let $l(x y)$ denote the arc length of the orbit between $x$ and $y$, using the metric induced by the Euclidean metric. Then put

$$
\begin{aligned}
l\left(a_{X} S_{X}\right)=\alpha & l\left(a_{Y} S_{Y}\right)=\hat{\alpha} \\
l\left(S_{X} t_{X}\right)=\beta & l\left(S_{Y} t_{Y}\right)=\hat{\beta} \\
l\left(S_{X} P_{X}\right)=\gamma & l\left(S_{Y} P_{Y}\right)=\hat{\gamma} \\
l\left(b_{X} S_{X}\right)=\delta & l\left(b_{Y} S_{Y}\right)=\hat{\delta}
\end{aligned}
$$

(see figure 9). Let $x \in \Sigma$ be close to, and below, $a_{X}$. Then the arc of orbit $x f_{X}(x)$ is
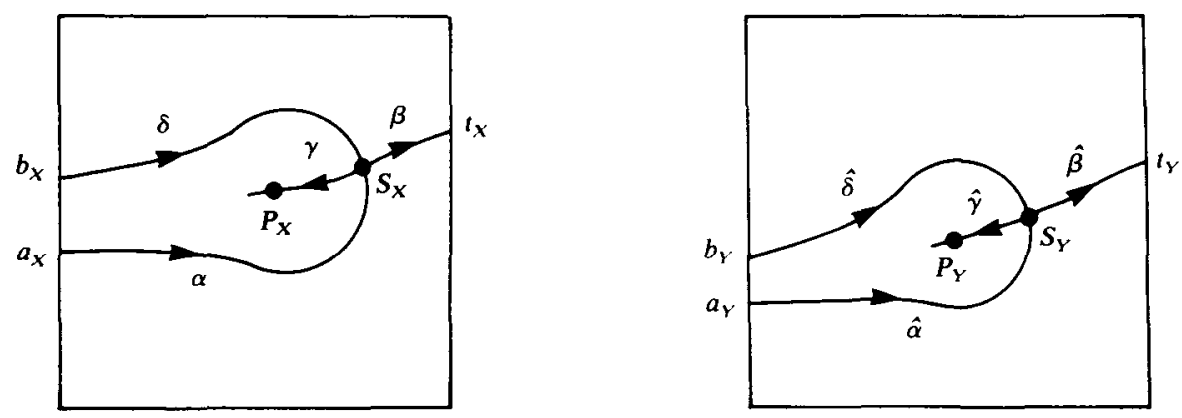

FIGURE 9

mapped onto an arc $y f_{Y}(y)$ determined by the map on $\Sigma$. Let $l\left(x f_{X}(x)\right)=r$ and $l\left(y f_{Y}(y)\right)=s$. We split $x f_{X}(x)$ into two parts of length $r \alpha /(\alpha+\beta)$ and $r \beta /(\alpha+\beta)$ and $y f_{Y}(y)$ into two parts of length $s \hat{\alpha} /(\hat{\alpha}+\hat{\beta})$ and $s \hat{\beta} /(\hat{\alpha}+\hat{\beta})$. The first and second parts of $x f_{X}(x)$ are then mapped onto the corresponding parts of $y f_{Y}(y)$ according to ratio of arc length. For points close to, and above, $a_{X}$ we do a similar procedure, this time splitting into parts of ratio $\alpha /(\alpha+\gamma)$ and $\gamma /(\alpha+\gamma)$ for $X$ and of ratio $\hat{\alpha} /(\hat{\alpha}+\hat{\gamma})$ and $\hat{\gamma} /(\hat{\alpha}+\hat{\gamma})$ for $Y$. We then do a similar procedure for points close to $b_{X}$. Finally for points away from $a_{X}$ and $b_{X}$ we map arcs of trajectories according 
to their whole lengths between intersections of $\Sigma$ and smooth these separate maps together by a partition of unity subordinate to a suitable cover of $\Sigma$.

Recall now the path $\phi \in C^{1}(I, \mathcal{N})$ examined in $\S 2$ and defined so that $f_{\phi(t)}=f_{\phi(0)}+t . \phi$ intersects each submanifold of Cherry fields or fields with a saddle connection at exactly one point. By the above remarks, to show that $\phi$ is mildly stable it suffices to show that a nearby path also meets every submanifold exactly once. This is true because $\phi$ intersects every submanifold transversely and furthermore when these submanifolds are close, they are $C^{\prime}$-close. Precisely, recall the maps $g_{\alpha}: \mathcal{N} \rightarrow S^{1}$ defined in $\S 2$ for any $\alpha \in[0,1) . g_{\alpha}$ takes the submanifold with rotation number $\alpha$ onto 0 (for $\alpha$ rational, $g_{\alpha}$ is really two maps). These maps tell us how much to add to the Poincaré map of the field to get the right rotation number $\alpha$. Then it follows that $g_{\alpha} \circ \phi=-\mathrm{id}+K_{\alpha}$ where $K_{\alpha}=\phi^{-1}\left(g_{\alpha}^{-1}(0)\right)$. So in particular this shows $\phi$ is transverse to the submanifold $g_{\alpha}^{-1}(0)$. Furthermore, as already noted if $g_{\alpha}$ and $g_{\beta}$ are close in $C^{0}$ sense, they are also close in the $C^{1}$ sense. Hence if $t_{0}=\left(g_{\alpha} \circ \phi\right)^{-1}(0)$ there are neighbourhoods $V_{\alpha}$ of $\phi$ in $C^{1}(I, \mathcal{N})$ and $U_{\alpha}$ of $t_{0}$ in $I$ such that if $\tilde{\phi} \in V_{\alpha}$ then $\tilde{\phi}$ intersects exactly once the same submanifolds as $\phi$ does, for $t \in U_{\alpha}$. We may find such a $U_{\alpha}$ for every $t \in E$, the bifurcation set of $\phi$ and then take a finite cover of $E$ by $U_{\alpha}$ 's, $\left\{U_{1}, \ldots, U_{n}\right\}$. Then $V=\bigcap_{i=1}^{n} V_{i}$ is a neighbourhood of $\phi$ such that $\tilde{\phi} \in V$ crosses every submanifold exactly once. Thus $\phi$ is mildly stable.

We now show that the topological equivalence changes continuously with $t$. First note that it is sufficient to do this on the restriction to $\Sigma$. So choose $\tilde{\phi} \in V$. We reparametrize $\tilde{\phi}$ by mapping the parameter linearly between corresponding bifurcation points. Let $q_{t}$ be the topological equivalence between $\phi(t)$ and $\tilde{\phi}(h(t))$, where $h$ is this reparametrizing homeomorphism. Fix $t_{0} \in I$. Let $I_{1}, I_{2}, \ldots$, and $\tilde{I}_{1}, \tilde{I}_{2}, \ldots$ be the successive inverse intersections with $\Sigma$ of the stable manifolds of $\phi\left(t_{0}\right)$ and $\tilde{\phi}\left(h\left(t_{0}\right)\right)$ respectively. Again, these 'intervals' may have two components if $\phi\left(t_{0}\right)$ is Morse-Smale. Let $\varepsilon>0$. We consider the three cases:

(a) $\phi\left(t_{0}\right)$ and $\tilde{\phi}\left(h\left(t_{0}\right)\right)$ are Cherry fields. Choose $N$ so large that $I_{1}, \ldots, I_{N}$ and $\tilde{I}_{1}, \ldots, \tilde{I}_{N}$ both have total lengths at least $1-\varepsilon / 4$. Then choose $\delta$ so small that if $\left|t-t_{0}\right|<\delta$, the corresponding intervals for $\phi(t), I_{1, t}, \ldots, I_{n, t}$, remain disjoint and let

$$
M=\sup _{\substack{\left|t-t_{0}\right|<\delta \\ 1 \leq j \leq N}} \frac{\left|\tilde{I}_{t, j}\right|}{\left|I_{t, j}\right|} .
$$

Then choose $\eta<\delta$ so small that the boundary points of $I_{t, 1}, \ldots, I_{t, N}$ and $\tilde{I}_{t, 1}, \ldots, \tilde{I}_{t, N}$ do not change by more than $\varepsilon / 4 M$ while $\left|t-t_{0}\right|<\eta$. Then $\left|t-t_{0}\right|<\eta \Rightarrow\left\|q_{t_{0}}-q_{t}\right\|<\varepsilon$ (see below).

(b) $\phi\left(t_{0}\right)$ and $\tilde{\phi}\left(h\left(t_{0}\right)\right)$ are Morse-Smale. Choose $N$ as in case (a). Choose $\delta$ so small that if $\left|t-t_{0}\right|<\delta$ then $\phi(t)$ is in the same Morse-Smale class. Put

$$
M=\sup _{\substack{t-t_{0} \mid<\delta / 2 \\ t \leq j \leq N}} \frac{\left|\tilde{I}_{t, j}\right|}{\left|I_{t, j}\right|}
$$

and choose $\eta<\delta / 2$ so small that for $\left|t-t_{0}\right|<\eta$ the endpoints of $I_{t, 1}, \ldots, I_{t, N}$ and $\tilde{I}_{t, 1}, \ldots, \tilde{I}_{i, N}$ do not change by more than $\varepsilon / 4 M$. Then $\left|t-t_{0}\right|<\eta \Rightarrow\left\|q_{t_{0}}-q_{t}\right\|<\varepsilon$ (see below). 
(c) $\phi\left(t_{0}\right)$ and $\phi\left(h\left(t_{0}\right)\right)$ have saddle connections. Choose $N$ and $M$ as in case (a). $\phi\left(t_{0}\right)$ is on the boundary of a certain Morse-Smale class. For $t<t_{0}$ suppose $\phi(t)$ fails to fall into this class. Then we may choose $\delta$ so small that for $\left|t-t_{0}\right|<\delta$, $I_{t, 1}, I_{t, 2}, \ldots, I_{t, N}$ and $\tilde{I}_{t, 1}, \ldots, \tilde{I}_{t, N}$ remain disjoint and their endpoints move by no more than $\varepsilon / 4 M$. On the other hand, for $t>t_{0}, \phi(t)$ is in the Morse-Smale class. Choose $\gamma$ so that for $\left|t-t_{0}\right|<\gamma$ the large components of $I_{t, 1}, \ldots, I_{t, N}$ and $\tilde{I}_{t, 1}, \ldots, \tilde{I}_{t, N}$ still have total length not less than $1-\varepsilon / 2$, and their endpoints move by no more than $\varepsilon / 4 M$. Put $\eta=\min (\delta, \gamma)$. Then $\left|t-t_{0}\right|<\eta \Rightarrow\left\|q_{t_{0}}-q_{t}\right\|<\varepsilon$.

To see this final step in each case, consider any $x \in \Sigma$. It must satisfy one of the following:

(i) $x$ remains outside any interval. Then $g_{t}(x)$ is outside any interval so is constrained to move by not more than $\varepsilon / 4+\varepsilon / 4 M$ in case (a) or (b), or $\varepsilon / 2+\varepsilon / 4 M$ in case (c).

(ii) $x$ remains inside a single interval. Consider figure 10 . The graph of $q_{t}$ for $\left|t-t_{0}\right|<\eta$ connects two points in the boxes. It is not hard to see that for the worst possible $x$ we have

$$
\left|q_{t}(x)-q_{t_{0}}(x)\right|<2 \cdot \varepsilon / 4 M+M \cdot \varepsilon / 4 M<\varepsilon .
$$

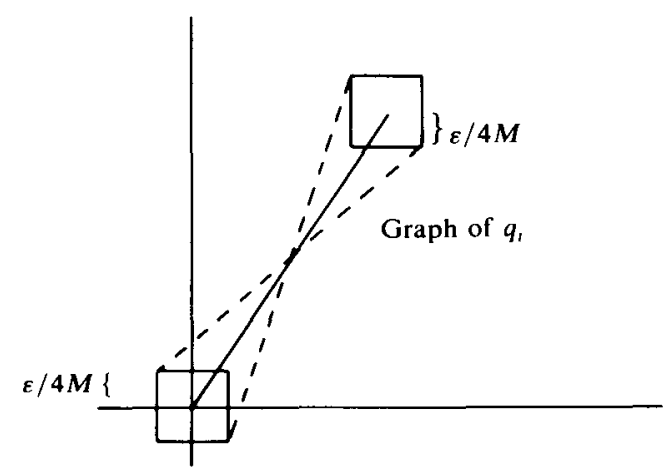

FIGURE 10

(iii) The endpoint of an interval crosses $x$. Call $e_{t}$ the endpoint directly below $x$ and suppose it was above $x$ for $t=t_{0}$. Then $d\left(x, e_{t_{0}}\right)<\varepsilon / 4 M$ and $d\left(x, e_{i}\right)<\varepsilon / 4 M$. In cases (a) or (b) we have

$$
d\left(q_{t_{0}}(x), \tilde{e}_{t_{0}}\right)<M \cdot \varepsilon / 4 M+\varepsilon / 4 \text { and } d\left(q_{t}(x), \tilde{e}_{t}\right)<\varepsilon / 4
$$

where $\tilde{e}_{t}$ is the corresponding endpoint for $\tilde{\phi}(t)$. Since we also have $d\left(e_{t_{0}}, e_{t}\right)<\varepsilon / 4 M$ it follows that

$$
d\left(q_{t_{0}}(x), q_{t}(x)\right)<\varepsilon / 2+\varepsilon / 4+\varepsilon / 4 M \leq \varepsilon
$$

(see figure 11). In case (c) the same argument holds, except we may need to replace $\varepsilon / 4$ by $\varepsilon / 2$ in one place.

Thus we have shown that $q_{t}$ is continuous in $t$ and so $\phi$ is a stable path as claimed. 

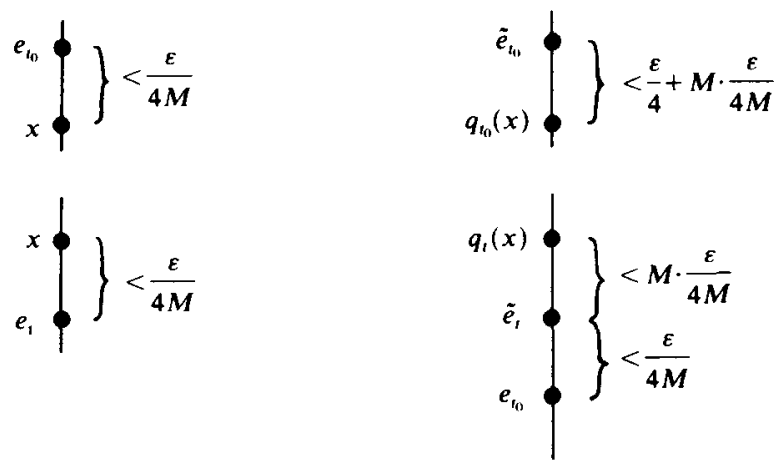

FIGURE 11

Acknowledgements. It is a pleasure to thank my supervisor Anthony Manning for help and advice during all stages of the preparation of this paper. I would also like to thank Mark Pollicott for many helpful conversations, and the SERC for financial support. This work was done while the author was at the University of Warwick.

\section{REFERENCES}

[1] V. I. Arnold. Small denominators, I. Mappings of the circumference onto itself. AMS Translations, Series 2, 46, 213-284.

[2] A. S. Besicovitch \& S. J. Taylor. On the complementary intervals of a linear closed set of zero Lebesgue measure. J. London Math. Soc. 29 (1954), 449-459.

[3] T. M. Cherry. Analytic quasi-periodic curves of discontinuous type on a torus. Proc. London Math. Soc. 44 (1938), 175-215.

[4] J. Dieudonné. Foundations of Modern Analysis. Academic Press, 1969.

[5] M. Herman. Mesure de Lebesgue et nombre de rotation. In: Geometry and Topology Rio de Janeiro 1976. Lecture Notes in Mathematics vol. 597, Springer-Verlag, 1977.

[6] M. W. Hirsch, C. C. Pugh \& M. Shub. Invariant Manifolds. Lecture Notes in Mathematics vol. 583. Springer-Verlag, 1977.

[7] W. Hurewicz \& H. Wallman. Dimension Theory. Princeton, 1941.

[8] J. Palis Jr. \& W. de Melo. Geometric Theory of Dynamical Systems. Springer-Verlag, 1982.

[9] G. R. Sell. Smooth linearization near a fixed point. Preprint, Minnesota, 1983.

[10] J. Sotomayor. Generic one parameter families of vector fields on two-dimensional manifolds. Pub. Math. Inst. Hautes Études Scientifiques, 43 (1973), 6-46.

[11] S. J. van Strien. One parameter families of vector fields. Thesis, Utrecht, 1982.

[12] S. J. Taylor. Introduction to Measure and Integration. Cambridge University Press, 1966. 